\title{
Introduction to the RE'09 special issue
}

\section{Kevin Ryan}

Published online: 20 May 2010

(C) Springer-Verlag London Limited 2010

\section{Editor's Introduction}

Each year since 1993, the Requirements Engineering research and practice community gather for the IEEE International Requirements Engineering conference (see http://www.requirements-engineering.org). It has become a tradition that a selection of the best research papers is identified and the authors invited to submit revised and enlarged versions of their papers for a special issue of the Requirements Engineering Journal. This is very appropriate since the REJ is the leading specialist publication in the field and, over the past few years, has become one of the most-cited publications in the broad field of software engineering. In September 2009, the 17th RE conference in the series was held in Atlanta, Georgia, in the USA. The conference theme was 'Requirements Engineering: the essential bridge'. To quote the call for papers

The RE research field builds the effective bridges between ... sub-disciplines of the Computer Science and Information Systems fields. The many computerbased system needs of business and society are often contradictory, inadequately defined, and rapidly changing. RE builds bridges between stakeholders, helping to reconcile their conflicts, clarify their goals, and reflect their priorities. If our society is to build a bridge to a better future we will need all of the models, methods, and tools that RE can provide.

Despite the economic situation which limited travel and training budgets the conference attracted over 200

K. Ryan ( $\square)$

Lero, The Irish Software Engineering Research Centre,

University of Limerick, Limerick, Ireland

e-mail: kevin.ryan@1ero.ie delegates from more than 25 countries. No doubt they were attracted by the four keynote speakers who reflected their interests in both research frontiers and industrial application. From industry, Dave West of Forrester Research spoke of how agile RE is delivering business value while Daryl Plummer of Gartner looked ahead to the RE challenges of cloud computing. Jim Herbsleb of Carnegie Mellon University widened the challenge by questioning the very possibility of a discipline of requirements. The fourth keynote, and it was an innovation to have four, was Alistair Sutcliffe of the University of Manchester who rounded off the conference with a reflection on the fundamental theories needed to bridge the 'gulfs' between people and machines. The conference was a balanced blend of such high-profile talks, technical presentations, industrial experience reports with a small number of lively panels and mini-tutorials. The RE week was rounded out by the collocated workshops and tutorials.

Of the 121 research papers submitted, only 21 were accepted as full papers. From this already select number, we have invited the authors of five of the very best papers to provide revised and expanded submissions for possible inclusion in this special issue. The papers have, as usual, been blind reviewed and all have been revised and improved until the reviewers judged each of them to be good enough for inclusion. Members of the RE'09 Program Committee as well as external experts served as reviewers. Together, the five papers represent a fascinating sample of the reach and depth of RE research in the first decade of the twenty-first century.

"Visual Syntax Does Matter: Improving the Cognitive Effectiveness of the i* Visual Notation" by Daniel Moody, Patrick Heymans and Raimundas Matulevicius is an expanded version of the paper which won the Best Paper award at RE'09. The authors take a radical perspective on 
the use of visual notations in requirements engineering in general and the widely used $i^{*}$ notation in particular. Using a principle-based approach, they perform a symbol by symbol critique of the $i^{*}$ notation and, where they identify deficiencies, they make preliminary suggestions for improvement.

With the increasing social reliance on large software systems has come the need to have systems that can modify themselves safely and efficiently, notwithstanding their inherent uncertainty. Expressing that uncertainty in wellfounded notation is the objective of "RELAX: A Language to Address Uncertainty in Self-Adaptive Systems Requirements" by Jon Whittle, Pete Sawyer, Nelly Bencomo, Betty H.C. Cheng and Jean-Michel Bruel. An example application to a smart home system is used to illustrate the approach and language. Remarkably, the lead author on this paper, Jon Whittle, is also an author on the next one.

A long-standing drawback of aspect-oriented and similar approaches is tackled by Gunter Mussbacher, Jon Whittle and Daniel Amyot in their paper "Modeling and Detecting Semantic-Based Interactions in Aspect-Oriented Scenarios". Their lightweight semantic approach aims to improve on existing methods that are purely syntactic but without requiring the full rigour of formalisation. One of the case studies used to demonstrate the application of their approach is an established challenge problem in the aspectoriented modelling community.

There has been a welcome increase in empirical studies within the RE community of late and the paper of James A. Miller, Remo Ferrari and Nazim H. Madhavji is a good example of the genre. "A controlled experiment to assess the impact of system architectures on new system requirements" will be valuable for both its methods and its results. They show that access to a system architecture has a significant impact on the number and type of requirements that are elicited by an analyst and they draw lessons for the research agenda in areas including traceability, processes and tools.

The final paper, "Deconstructing the Semantics of BigStep Modelling Languages" is by Shahram Esmaeilsabzali, Nancy A. Day, Joanne M. Atlee, David R. Cheriton and Jianwei Niu. With a view to characterising some of the numerous modelling languages that are used in RE, the authors define the class of Big-Step Modelling Languages (BSMLs) and investigate their semantics and the relative advantages these confer on them. The resulting tables can assist an analyst in their choice of BSML.

It is the welcome task of the RE Programme Chair to draw together the special issue of the REJ. However, it requires the selfless and effective work of a team of people before the issue becomes a reality. In the current case, I would like to acknowledge the advice and guidance of Martin Glinz, then Chairman of the RE Conference Steering Committee (and Program Chair of RE'06), the support of Peri Loucopoulos and his REJ editorial team and, especially, the timely and professional work of the anonymous reviewers. None of this would have been possible without the outstanding work of Bill Robinson, the General Chair of RE'09.

I trust this special issue will give pleasure and inspiration to those who read it. I hope it will encourage new researchers to pursue the never-ending quest for the improved RE theory and practice that will serve society and commerce in the years ahead. The best place to keep yourself up to date is, as ever, in the REJ and at the RE conferences, in Sydney (2010) and in Trento (2011). 